\title{
Silicon PIN photodiode-based radon detectors for an underground experiment environment.
}

\author{
Kyungmin Seo ${ }^{1}$, Hyunsoo Kim \\ Department of Physics, Sejong Univercity \\ 209 Neungdong-ro, Gwangjin-gu, Seoul 05006, Korea \\ E-mail: sukm2000 at gmail.com
}

Hyeyoung Lee, Moo Hyun Lee, Wootae Kim, Yeongduk Kim

Center for Underground Physics, Institute for Basic Science (IBS)

55 Expo-ro, Yuseong-gu, Daejeon 34126, Korea

\begin{abstract}
It is very important to monitor the amount of radon $\left({ }^{222} \mathrm{Rn}\right)$ in the underground experiments such as rare decay search and dark matter experiments with ultra-low background requirements. The radioactivity from the radon can be a significant background source to the experiments and need to be measured precisely. We upgraded a radon detector with a volume of $\sim 70 \mathrm{~L}$ which was used in the KIMS (Korean Invisible Matter Search) experiment by replacing a Hamamatsu silicon PIN photodiode and a Hamamatsu pre-amplifier. The positively charged radon's daughter particles $\left({ }^{214} \mathrm{Po}\right.$ and ${ }^{218} \mathrm{Po}$ mostly) produced in the air of the detector chamber are collected by the photodiode in a negative high voltage. The energy resolutions of alpha particles emitted from the decays of the daughter particles are measured to be $\sim 0.3 \%$ with very clean signals to be identified. In this presentation, we also made two more radon chamber detectors and have been testing them to be used in the underground experiment facility. We will present performances of the radon chamber detectors in the tests.
\end{abstract}

The 39th International Conference on High Energy Physics (ICHEP2018)

4-11 July, 2018

COEX, Seoul, Korea

\section{${ }^{1}$ Speaker}




\section{Introduction}

Radon is an inert gas and has colorless, tasteless and odorless properties. It is also heavier than air. During the process of natural decay of uranium or thorium which is common on the earth, the radon is produced in the decay chain. Because the radon itself is an inert gas, it can easily penetrate into the detector. Since there are many radioactive backgrounds from the radon deposited inside the detector, it is very important for the underground experiment to monitor the radon concentration in the environment. To measure the concentration of radon, we use polonium, the daughter isotopes of radon. They are positively charged and can be easily collected by using negatively charged electric field.

\section{Radon chamber}

We upgraded a radon chamber detector with a volume of $\sim 70 \mathrm{~L}$ which was used in the KIMS (Korean Invisible Matter Search) experiment by replacing with a Hamamatsu silicon PIN photodiode and a Hamamatsu pre-amplifier. After confirming an improvement in energy resolution of the upgraded detector, two new chamber detectors were produced as shown in Fig. 1. Polonium produced by the decay of radon is attached to the silicon photodiode after drifting along the formed electric field of $-1000 \mathrm{~V}$. When alpha particles released from the polonium decays are directing toward the silicon diode, we can measure the signals clearly.

Three peaks from the alphas released by decays of polonium can be seen in the peak height distribution as shown in Fig. 2. The peaks were fitted using a crystal ball function. A sigma

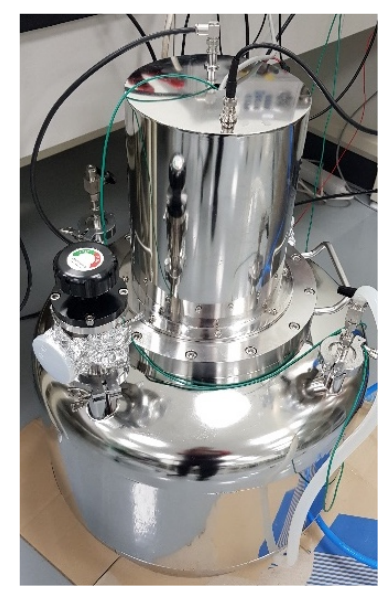

Figure 1: One of two newly built radon chamber detectors.

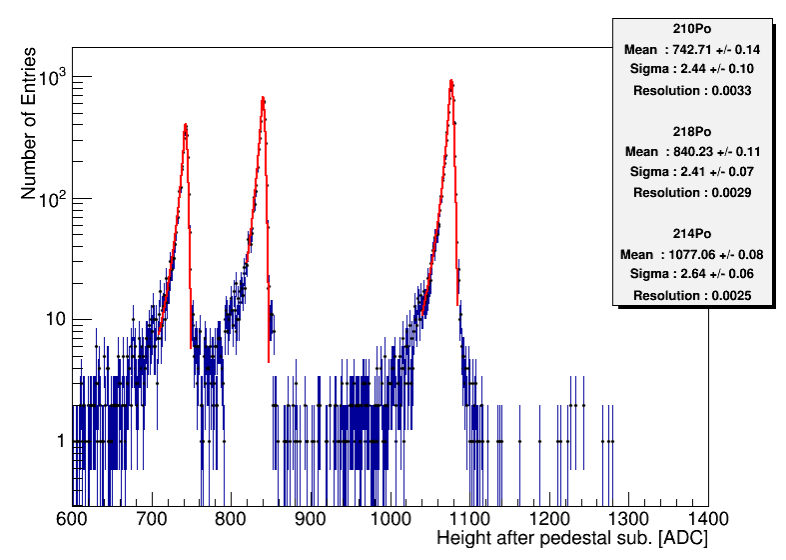

Figure 2: A pulse height distribution from the detector. Three alpha peaks from ${ }^{210} \mathrm{Po},{ }^{218} \mathrm{Po}$, and ${ }^{214} \mathrm{Po}$ decays are clearly seen.

resolution of $\sim 0.3 \%$ was obtained for each of the peaks. The three peaks show a good linearity in energy response of the silicon sensor.

A $~ 22$ days of long-term test after flushing $\mathrm{N}_{2}$ gas showed a slight decrease of polonium with time, which indicates no outside gas leakage into the chamber.

\section{References}

[1] M.J. Lee et al., Radon Environment in the Korea Invisible Mass Search Experiment and Its Measurement, J. Korean Phy. Soc. 58 (2011) 713.

[2] C. Mitsuda et al., Development of super-high sensitivity radon detector for the Super-Kamiokande detector, Nucl. Instrum. Methods Phys. Res., Sect. A 497 (2003) 414. 